\title{
Ação educativa para as gestantes na promoção da "Golden hour": relato de experiência
}

\author{
Educational activity for pregnants in the promotion of Golden hour: experience report
}

Acción educativa para el mujeres embarazadas en la promoción de la "Hora Dorada": relato de experiencia

Rafaela Pereira de Sena ${ }^{1 *}$, Gabriela Nascimento de Souza ${ }^{1}$, Luiza Alessandra Oliveira Monteiro', Yenne Patrícia Rodrigues de Souza', Yves de Luka Miranda dos Santos ${ }^{1}$, Maria Rute de Souza Araujo ${ }^{1}$, Silvia Cristina Santos da Silva ${ }^{1}$, Rodrigo Maia da Silva ${ }^{1}$, Jorgeany Soares Parente ${ }^{1}$.

\section{RESUMO}

Objetivo: Relatar a experiência vivenciada pela atividade educativa para gestantes na promoção da Golden hour, realizada em uma Estratégia Saúde da Família (ESF), no município de Belém-PA. Relato da experiência: Foi realizada uma roda de conversa no qual abordou-se o tema "Golden hour", enfatizando sua importância na vida do recém-nascido e da mãe, de forma crítica-reflexiva observou-se a visão e o interesse que as mesmas manifestaram sobre tais assuntos. O uso das metodologias ativas permitiu as gestantes aquisição de novos conhecimentos sobre a temática e um repensar da importância de saber sobre seus direitos e ainda com uso de dinâmicas envolvendo músicas, balões e perguntas relacionadas ao assunto desenvolvido, foi percebido carências de informações e orientações dos profissionais de saúde para com as gestantes sobre a importância do tema. Além disso, para esse estudo foi disponibilizado tecnologias em forma de folhetos educativos, no qual continham orientações e conhecimentos acerca da hora de ouro. Considerações Finais: É necessário o empoderamento feminino para garantir esses direitos, assim como a sensibilização dessas mulheres pelos profissionais de saúde afim de reduzir a morbimortalidade neonatal seguindo os cuidados corretos das boas práticas desta primeira hora.

Palavras-chave: Gestantes, Profissionais de saúde, Empoderamento.

\section{ABSTRACT}

Objective: Relate the experience experienced by the educational activity for pregnants in the promotion of Golden Hour, realized in a Family Health Strategy (FHS), in the city of Belém-PA. Experience report: It was realized a conversation round where it was approached the theme "Golden hour", emphasizing the importance in the life of the newborn and the mother, in a critical-reflexive way it was observed the vision and the interest that they expressed on such subjects. The use of active methodologies allowed pregnants to acquire new knowledge about the theme and a rethink of the importance of knowing about their rights and also with the use of dynamics involving music, balloons and questions about the developed subject, it was perceived lack of information and guidance from health professionals to the pregnants about the importance of the theme. In addition, for this study, it was available technologies in the form of educational leaflets, which contained guidelines and knowledge about the golden hour. Final Considerations: It is necessary the female empowerment to guarantee these rights, as well the sensitization of these women by the health professionals in order to reduce the neonatal morbidity and mortality following the correct care of good practices of this first hour.

Key words: Pregnants, Health professionals, Empowerment.

${ }^{1}$ Centro Universitário Metropolitano da Amazônia (UNIFAMAZ), Belém-Pará

*E-mail: rafaelapsena12@gmail.com

SUBMETIDO EM: 11/2019

ACEITO EM: 12/2019

PUBLICADO EM: 2/2020

REAS/EJCH | Vol.Sup.n.40 | e2291 | DOI: https://doi.org/10.25248/reas.e2291.2020 Página 1 de 6 


\section{RESUMEN}

Objetivo: Informar sobre la experiencia vivida por la actividad educativa para mujeres embrazadas en la promoción de la Hora Dorada, realizada en una Estrategia de Salud Familiar (ESF), en la ciudad de BelémPA. Informe de la experiencia: Se llevó a cabo una ronda de conversación en la que se abordó el tema "La hora dorada", enfatizando su importancia en la vida del recién nacido y la madre. De una manera críticareflexiva, se observó la opinión y el interés que expresaron sobre tales temas. El uso de metodologías activas permitió a las mujeres embarazadas adquirir nuevos conocimientos sobre el tema y repensar la importancia de conocer sus derechos y también con el uso de dinámicas que involucran canciones, globos y preguntas relacionadas con el tema desarrollado. La orientación de los profesionales de la salud a las mujeres embarazadas sobre la importancia del tema, y para este estudio, se proporcionaron tecnologías en forma de folletos educativos, que contenían orientación y conocimiento sobre la hora dorada. Consideraciones Finales: El empoderamento feminino es necesario para garantizar estos derechos, así como la sensibilización de estas mujeres por parte de los profesionales de la salud para reducir la morbilidad y mortalidad neonatal después del cuidado correcto de las buenas prácticas de esta primera hora.

Palabras clave: Mujeres embarazadas, Profesionales de la salud, Empoderamiento.

\section{INTRODUÇÃO}

Os primeiros 60 minutos de vida do bebê após o nascimento, chamado de Golden hour ou Hora de ouro, representam o período no qual são realizadas intervenções para minimizar as complicações neonatais. Dentre tais intervenções, o contato pele a pele e a amamentação na primeira hora de vida são práticas extremamente imprescindíveis para o estabelecimento do vínculo mãe e bebê, além de prolongar a duração do aleitamento materno (ARRUDA GT, et al., 2018).

Embora haja a ampliação da cobertura do acompanhamento do pré-natal no país e consequentemente redução da mortalidade materna, nas últimas décadas, o número de óbitos neonatais continua insatisfatório, devido as complicações que ocorrem nesse período, sendo que muitos desses óbitos ocorrem por causas evitáveis, podendo ser minimizados durante um bom cuidado pré-natal (OLIVEIRA EC, et., 2016). O pré-natal bem realizado não apenas reduz complicações durante a gestação, mas também facilita a atuação dos especialistas na sala de parto, assim diminuindo as infeções e os riscos iminentes do parto, sendo um procedimento totalmente possível de ser bem realizado por médicos e enfermeiros, com padrão de alta qualidade (DIAS RA, 2014).

O Aleitamento Materno (AM) na primeira hora de vida é reconhecido pela Organização Mundial da Saúde (OMS) como um componente importante na promoção, proteção e suporte, podendo ser implementado como prática hospitalar rotineira em todos os países, reduzindo a mortalidade neonatal (SOCIEDADE BRASILEIRA DE PEDIATRIA, 2013). De acordo com a OMS, 33\% dos recém-nascidos que não foram direto para o peito de suas mães na primeira hora de vida apresentaram maior risco de mortalidade, do que os que receberam o AM. Os bebês que são amamentados ficam menos doentes e são mais nutridos do que aqueles que ingerem qualquer outro tipo de alimento. Sendo que o AM deve ser exclusivo até os seis meses de idade.

Além de ser imprescindível para a saúde da criança o AM apresenta inúmeras vantagens para a saúde das mães, ajudando-as na recuperação pós-parto, diminuindo o risco de hemorragias, ajudando-as voltar ao peso anterior a gestação, reduzindo o risco de câncer de mama e de ovário (ARAÚJO AL, 2018). O enfermeiro tem um papel fundamental no AM, tendo como função orientar, desempenhar o desenvolvimento da promoção e apoio a amamentação, com objetivo de incentivar e sensibilizar o desmame tardio (ARAÚJO AL, 2018).

Ao considerar tais benefícios, preconiza-se que a separação da mãe e do recém-nascido (RN) nas primeiras horas de vida seja evitada, favorecendo o contato pele a pele, pois tal prática auxilia em vários mecanismos de autorregulação do RN, dentre eles a manutenção da temperatura corporal constante, ajuda na expulsão da placenta e incentiva o vínculo entre mãe e filho (RUSCHEL LM, et al., 2018).

Uma outra prática da Golden hour é o clampeamento oportuno do cordão umbilical, pois além da adaptação fisiológica do recém-nascido à respiração extrauterina, o atraso do clampeamento entre 30 a 180 
segundos após o nascimento proporciona um aumento dos níveis de hematócrito e hemoglobina, reduzindo o risco de anemia ferropriva nos primeiros 4 a 6 meses de vida (GOES JF, 2017).

Portanto, o momento ideal para pinçar o cordão de todos os recém-nascidos, independentemente de sua idade gestacional, é quando a circulação do cordão umbilical cessar (permanecer achatada e sem pulso), aproximadamente 3 minutos ou mais após o nascimento, em seguida procede-se o clampeamento e o corte (BRASIL, 2011). O papel do enfermeiro deve ser desenvolvido e avaliado continuamente, visto que com ferramentas como educação em saúde requer um pensar crítico e reflexivo, para desenvolver atividades, permitindo observar a realidade e propor ações transformadoras que levem o indivíduo a sua autonomia e independência.

Com isso, a educação em saúde está relacionada à aprendizagem, podendo contribuir na formação de consciência crítica do educando, culminando na aquisição de práticas que visem à promoção de sua própria saúde e da comunidade na qual encontra-se inserido (PAES CCDC e PAIXÃO ANP, 2016).

Sendo assim, a motivação para desenvolver essa temática foi a verificação de que existe necessidade de proporcionar educação em saúde para o empoderamento das gestantes, no que tange as práticas realizadas com o RN na sala de parto. O objetivo deste estudo é relatar a experiência vivenciada na atividade educativa para gestantes na promoção da Golden hour, realizada em uma Estratégia Saúde da Família (ESF), no município de Belém-PA.

\section{RELATO DE EXPERIÊNCIA}

A ação educativa foi realizada em uma ESF, no município de Belém-PA, apresentando um total de 35 gestantes acompanhadas pelo enfermeiro da unidade, porém a atividade de educação em saúde, foi realizada com 7 grávidas antes da consulta de rotina, com idade entre 16 a 37 anos e idade gestacional entre 9 a 39 semanas, no turno vespertino, em outubro de 2019. Na abertura das atividades, foi realizada uma roda de conversa no qual foi abordado o tema "Golden hour", enfatizando sua importância na vida do recém-nascido e da mãe, de forma crítica-reflexiva, foi observado a visão e o interesse que as mesmas manifestaram sobre tais assuntos como: clampeamento oportuno do cordão umbilical, contato pele a pele imediato, amamentação exclusiva até os seis meses de idade, direitos e deveres da paciente e seus acompanhantes.

No início percebeu-se, as gestantes tímidas e retraídas com sensação de medo para não errar ou por não terem conhecimento dos assuntos abordados, pois apenas três gestantes já tinham conhecimento sobre os benefícios do aleitamento materno tanto para mãe quanto para o bebê e sendo uma prática exclusiva até os seis meses de idade, do vínculo que promove essa prática combinada com o contato pele a pele para 0 estreitamento afetivo de ambos. No entanto, sobre o clampeamento oportuno e sobre os direitos e deveres da paciente e seus acompanhantes houve mais dúvidas e falta de conhecimento. Os olhares atentos e o silêncio após as explicações, possibilitou entender que as gestantes pouco sabiam que podiam ter acesso a um parto mais humanizado e que podiam buscar mais informações sobre as práticas aplicadas na sala de parto, que possibilitam maior segurança para ela e para o bebê no momento do nascimento. Percebeu-se também, que a falta de conhecimento ou a inexperiência de serem primigestas, dificulta mais ainda o processo das boas práticas na sala de parto.

No segundo momento foi realizada a metodologia ativa (MA) com uso de dinâmicas envolvendo músicas, balões e perguntas relacionadas ao assunto desenvolvido, onde foi percebido carências de informações e orientações dos profissionais de saúde para com as gestantes sobre a importância desse tema. Além disso, para esse estudo foi disponibilizado tecnologias em forma de folhetos educativos, no qual continham orientações e conhecimentos acerca da hora de ouro (Figura 1).

As orientações contidas nos folhetos educativos eram sobre as boas práticas da hora de ouro, explicando os benefícios que elas trazem para o recém-nascido, como por exemplo o clampeamento oportuno previne a deficiência de ferro e a anemia durante a infância, o contato pele a pele ajuda a regular a temperatura do recém-nascido e fortalece o vínculo entre mãe e bebê e sobre o leite materno que é rico em nutrientes e previne contra doenças infecciosas. Assim os folhetos educativos serviram como suporte de levar mais um 
pouco de conhecimento da temática, tentando sensibilizar essas gestantes a busca sobre seus direitos e deveres, garantindo segurança para ela e para seu bebê no momento do parto.

No final percebeu- se, que o folheto educativo ajudou mais ainda no processo de entendimento dessas gestantes, pois todas guardaram os folhetos para lerem novamente em suas casas e algumas relataram que não sabiam o quão importante era, um simples ato de amamentar para vida de seus filhos. Neste sentido, a experiência vivenciada nesta ação educativa, foi de grande relevância tanto para as acadêmicas quanto para essas gestantes que se propuseram a adquirir um pouco mais de conhecimento acerca dessa temática.

Figura 1 - Folheto Educativo.

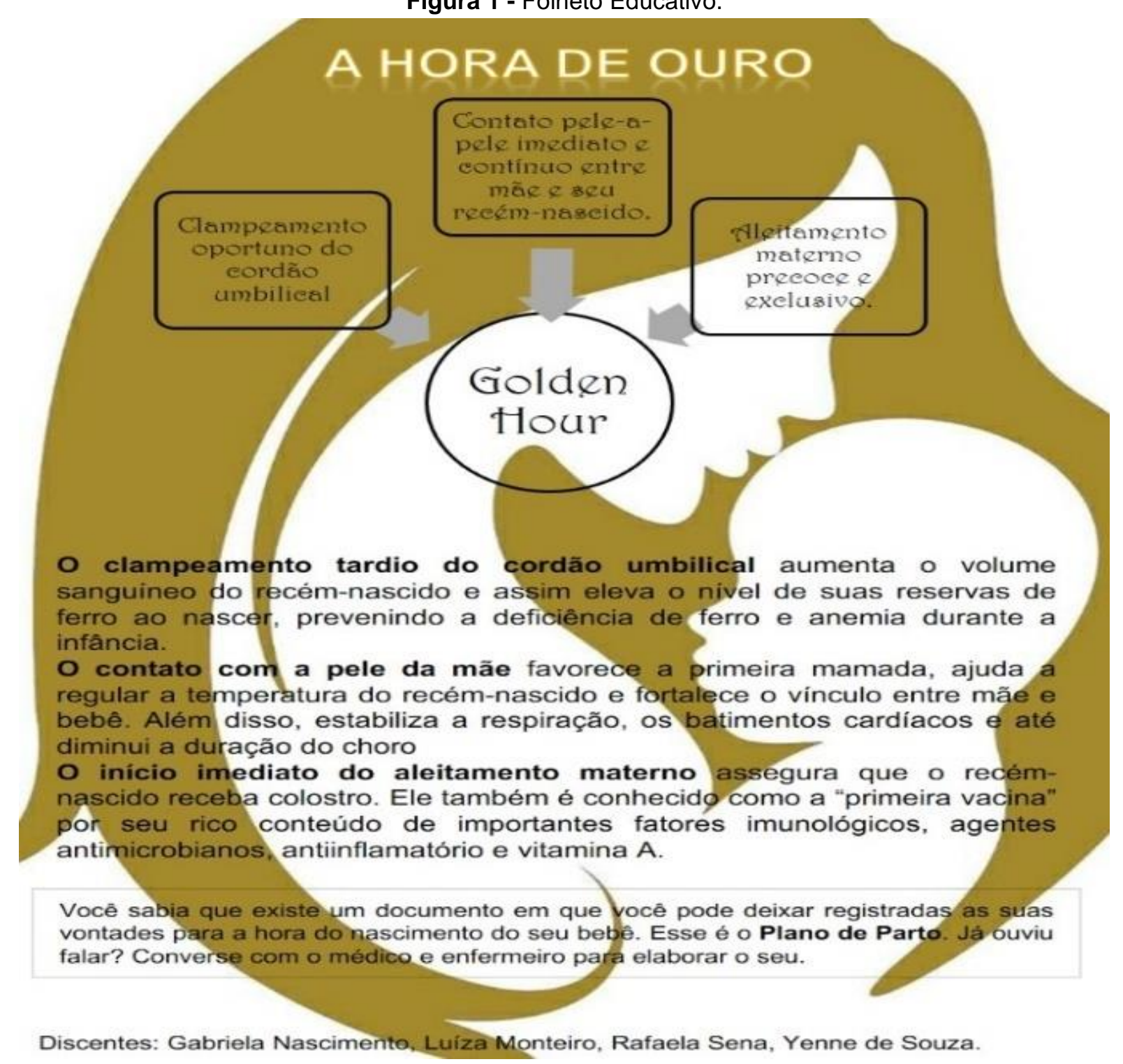

Fonte: Sena RP, et al., 2019.

A dinâmica composta por perguntas, possibilitou perceber as dificuldades encontradas diante das respostas exigidas, pois somente três gestantes já sabiam a importância do aleitamento materno exclusivo até os seis meses e já tinham ouvido falar sobre o contato pele a pele, mas sobre o clampeamento oporturno e dos direitos sobre um plano de parto, exigiu mais esforço por parte delas para encontrarem respostas corretas e o tempo exigido para as respostas foi maior do que o estabelecido no início da brincadeira. Houveram perguntas como: quais os benefícios que o clampeamento oportuno promove ao recém-nascido? Quantos minutos são indicados para o clampeamento do cordão umbilical? Quais os benefícios do 
aleitamento materno para a mãe o e bebê? e dentre outras. Assim, conforme cada acerto as vencedoras eram selecionadas para próxima jogada, restando apenas uma no final da brincadeira.

\section{DISCUSSÃO}

Foi realizada metodologia ativa (MA), pois ajuda o entendimento de forma mais simples gerando uma educação reflexiva com base nos estímulos do processo ensino-aprendizagem, resultando em envolvimento, mobilizando o educando a busca de conhecimento (MACEDO KDS, et al., 2018). A presença das tecnologias de informação e comunicação é cada vez mais forte e notória. A utilização dessas geram grandes mudanças na educação-saúde, onde se forem conduzidas e utilizadas de maneira correta, pode gerar benefício para a pessoa que está de certo modo ligada às transformações tecnológicas (OTTO PA, 2016).

O resultado encontrado no estudo revela que a gestação está associada a mudanças biopsicossociais que influenciam a vida individual e interpessoal da gestante. Além disto, a maneira como a gestante vive estas mudanças colabora intensamente no modelo da maternidade e na relação mãe-bebê. Portanto, vivenciam intensos sentimentos em relação ao tornar-se mãe e o processo de constituição da maternidade em fase de desenvolvimento (PICCININI CA, et al., 2008).

Nessa perspectiva, a inserção das tecnologias no cotidiano requer a reorganização dos serviços e o desenvolvimento de capacitações e competências dos profissionais sendo ferramentas essenciais para construção de novas práticas em saúde que auxiliem nessas modificações vivenciadas pela mulher. Essa reorganização do trabalho deve valorizar o acesso a informação, expansão e organização da rede para 0 cuidado progressivo e humanizado ao usuário (OLIVEIRA G, et al., 2017).

A estratégia pode ser utilizada durante o pré-natal e puerpério por permitir as gestantes uma participação ativa e a possibilidade de desenvolver sua autonomia e independência, já que na promoção da saúde, as práticas educativas devem contribuir, para o fortalecimento e capacidade de escolha. Assim, as informações devem ser repassadas de forma simples e contextualizadas fortalecendo o entendimento das mesmas (ALVES GG, et al., 2011).

Entre as participantes, cinco iniciaram o pré-natal no primeiro trimestre e apenas duas gestantes realizaram três consultas, sendo que as outras realizaram, em média, de 1 a 11 consultas. Nesse sentido, segundo Tomasi E, et al. (2017), a manutenção e a melhoria da saúde materno-neonato são alguns dos objetivos definidos pelo Ministério da Saúde e, para isto, é essencial a atenção pré-natal e puerperal, cuja responsabilidade é do Sistema Único de Saúde (SUS) onde é preconizado que este pré-natal se inicie com acompanhamento no primeiro trimestre gestacional e que sejam realizadas, no mínimo, seis consultas.

Dessa maneira, a assistência ao pré-natal, não deve se reduzir apenas à realização de consultas e solicitação de exames, pois precisa considerar também o acolhimento e o reconhecimento das necessidades das gestantes, visando o estabelecimento de vínculos e o emponderamento dessas mulheres (LIVRAMENTO DVP, et al., 2019).

Estudos revelam, que a equipe no momento do parto tem um olhar voltado para as necessidades do recém-nascido, realizando ações para a adaptação extrauterina, de forma mecânica e rotineira. Não valorizando e respeitando os sinais emitidos pela parturiente, reafirmando a visão da mulher submissa, responsável pela reprodução. Por isso o apoio profissional à parturiente é de suma importância para favorecer os sentimentos e benefícios da hora de ouro (SANTOS LM, et al., 2014).

Foi observado com a dinâmica e o folheto educativo que grande parte do grupo se interessou em saber e conhecer mais sobre o assunto, pois nenhuma das gestantes presentes conheciam o tema abordado, assim não identificando a importância de um momento tão único entre mãe e filho. Um dos questionamentos foi sobre as leis que amparam seus direitos e seus desejos respeitados na hora do parto. Com isso a educação em saúde foi de grande relevância, pois conseguiu-se alcançar o objetivo, levando acesso e conhecimento para essas futuras mamães contribuindo dessa maneira, com a formação dos conhecimentos adquiridos na ação educativa.

REAS/EJCH | Vol.Sup.n.40 | e2291 | DOI: https://doi.org/10.25248/reas.e2291.2020 Página 5 de 6 


\section{CONSIDERAÇÕES FINAIS}

Percebeu-se que embora haja intensa imersão neste cotidiano tecnológico, subsidiado pela era em que vivemos, com o fluxo continuo e ágil de informações, o tema "Golden hour" ainda é pouco conhecido pelas mulheres, dificultando assim os direitos destas, na busca por um parto humanizado. Por isso, é necessário o empoderamento feminino para garantir esses direitos, assim como a sensibilização dessas mulheres pelos profissionais de saúde afim de reduzir a morbimortalidade neonatal seguindo os cuidados corretos das boas práticas desta primeira hora.

\section{REFERÊNCIAS}

1. ALVES GG, AERTS D. As práticas educativas em saúde e a estratégia saúde da família. Ciência saúde coletiva. $2011 ; 16: 19-325$.

2. ARAÚJO AL. Cuidado de Enfermagem ao recém-nascido prematuro na UTI Neonatal. Trabalho de Conclusão de Curso (Enfermagem) - Universidade Anhanguera, Osasco/SO, 2018.

3. ARRUDA GT, et al. Existe relação da via de parto com a amamentação na primeira hora de vida? Revista Brasileira em Promoção da Saúde 31 (2), 2018.

4. DIAS RA. A importância do pré-natal na atenção básica. Nescon, UFMG, 2014

5. GOES JF. Clampeamento tardio do cordão umbilical: Estudo de corte. Rio de Janeiro 2017.

6. LIVRAMENTO DVP, et al. Percepções de gestantes acerca do cuidado pré-natal na atenção primária à saúde. Rev. Gaúcha Enfermagem, 2019; ISSN 0102-6933.

7. MACEDO KDS et al. Metodologias ativas de aprendizagem: Caminhos possíveis para inovação no ensino em saúde. Esc. Anna Nery, vol.22, n.3 2018.

8. OLIVEIRA EC et al, A importância do acompanhamento pré-natal realizado por enfermeiros. Revista Científica FacMais, Vol VII, Núm 3. 2016.

9. OLIVEIRA G, et al. Acesso de gestantes às tecnologias para prevenção e controle da sífilis congênita em Fortaleza - Ceará, Brasil. Revista de Salud Pública 2017; ISSN 0124-0064.

10. ONUBR: Três em cada cinco bebês não são amamentados na primeira hora de vida no mundo.

11. OTTO PA. A importância do uso das tecnologias nas salas de aula nas series iniciais do ensino fundamental I. Florianópolis - SC, 2016.

12. PAES CCDC, PAIXÃO ANPA. Importância da abordagem da educação em saúde: Revisão de Literatura. REVASF, Petrolina - PE, vol. 6, n.11, p. 80-90 dez. 2016.

13. PICCININI CA, et al. Gestação e a constituição da maternidade. Psicologia em Estudo, Maringá, v. 13, n. 1, p. 6372, jan./mar. 2008.

14. RUSCHEL LM, et al. Hipotermia e banho do recém-nascido nas primeiras horas de vida. Rev Gaúcha Enferm. 2018.

15. SANTOS LM, et al. Vivenciando o contato pele a pele com o recém-nascido no pós-parto como um ato mecânico. Rev. Bras. Enferm. 2014.

16. SOCIEDADE BRASILEIRA DE PEDIATRIA. Aleitamento materno na primeira hora de vida protege contra mortalidade neonatal, Vol. 89, Núm. 2, Março-Abril, 2013.

17. TOMASI E, et al. Qualidade da atenção pré-natal na rede básica de saúde do Brasil: Indicadores e desigualdades sociais. Cad. Saúde Pública. 2017. 